\title{
Publisher's Note: Sub GV/cm terahertz radiation from relativistic laser-solid interactions via coherent transition radiation [Phys. Rev. E 93, 063204 (2016)]
}

\author{
W. J. Ding (丁文君) and Z. M. Sheng (盛政明) \\ (Received 16 June 2016; published 23 June 2016)
}

DOI: 10.1103/PhysRevE.93.069903

This paper was published online on 13 June 2016 with a conversion error in Eq. (3). Equation (3) should read as

$$
\frac{d^{2} \mathcal{E}_{\mathrm{CTR}}}{d \omega d \Omega}=\frac{e^{2} N(N-1)}{\pi^{2} c}\left|\int S(\beta, \varphi, \phi) f(\tau, \boldsymbol{\rho}, \boldsymbol{v}) e^{i \omega \tau-i \boldsymbol{q} \cdot \boldsymbol{\rho}} d \tau d \boldsymbol{\rho} d \boldsymbol{v}\right|^{2}
$$

The equation has been corrected as of 16 June 2016. The equation is correct in the printed version of the journal. 